\title{
The Influence of Front Wing Pressure Distribution on Wheel Wake Aerodynamics of a F1 Car
}

\author{
Daniel Martins, João Correia and André Silva *(D) \\ Aerospace Sciences Department, University of Beira Interior, Calçada Fonte do Lameiro, 6201-001 Covilhã, \\ Portugal; daniel.martins@ubi.pt (D.M.); joao.correia@correiaracing.com (J.C.) \\ * Correspondence: andre@ubi.pt
}

Citation: Martins, D.; Correia, J.; Silva, A. The Influence of Front Wing Pressure Distribution on Wheel Wake Aerodynamics of a F1 Car. Energies 2021, 14, 4421. https://doi.org/ 10.3390/en14154421

Academic Editor:

Francesco Castellani

Received: 6 June 2021

Accepted: 16 July 2021

Published: 22 July 2021

Publisher's Note: MDPI stays neutral with regard to jurisdictional claims in published maps and institutional affiliations.

Copyright: (c) 2021 by the authors. Licensee MDPI, Basel, Switzerland. This article is an open access article distributed under the terms and conditions of the Creative Commons Attribution (CC BY) license (https:// creativecommons.org/licenses/by/ $4.0 /)$.

\begin{abstract}
The present study focuses on investigating the aerodynamic interaction between a threeelement wing and wheel in ground effect, following the Formula One regulation change set for 2022, among which is the simplification of the front wing. This was accomplished by conducting a three-dimensional computational fluid dynamics analysis, using a Detached-Eddy Simulation approach, on a simplified one-quarter model of a Formula One racing car. The main goal was to examine how changing the front wing pressure distribution, by changing the incidence of the second flap, affected the wheel wake. The flow investigation indicated that the wheel wake is influenced by the flap configuration, which is mainly due to the fact that different flap configurations produce different upwash flow fields, leading to a variation of the separation point on top of the tire. As the separation point moves rearwards, the downwash generated in the central region (for a vertical plane) of the wheel wake increases incrementally, leading to a resultant wake that is shorter and further apart. The force investigation showed that the proximity between the region of instability (i.e., vortex breakdown) and the wing's trailing edge influences the behavior of the transient oscillations, regarding the forces acting on the wing: detecting higher drag force fluctuations, when compared to downforce fluctuations.
\end{abstract}

Keywords: Formula One; racing car; front wing; wheel; transient; CFD

\section{Introduction}

The field of aerodynamics is one of the most important and impactful factors in the modern world of Formula One racing. Nowadays, teams invest a significant amount of their budget on the research and development of aerodynamics in the hopes of achieving considerable performance gains.

One of the governing rules of Formula One dictates that all four wheels of the car must be exposed. Such a requirement means that there is no bodywork around the wheels to control what is already a complicated flow field both around and downstream of the wheels. In addition, the front wheels are accountable for approximately $40 \%$ of the total drag of the car [1]. With that in mind, one can conclude that the flow around the front wheels is of extreme importance. Its mismanagement can result in regions of energy loss, having an impact on the performance of downstream components. The front wing of a Formula One racing car has a very important role in all of this. Being the first element to encounter the free stream of air, the front wing dictates the shape of the flow around the other components on the car. The front wing is responsible for generating a trailing vortex system, consisting of several co-rotating vortices [2], shed by the endplates, strakes, and the central section of the wing. These vortices then control the wake of the front wheels by re-directing it away from the car.

Concerning the near-wake of an isolated wheel, Mercker and Benerburg [3] named the structures observed to the side of the wheel, where the tire meets the ground, as "jetting vortices". These result from the strong viscous forces in front of the tire contact patch [4]. 
The phenomenon, called "tire jetting", happens because the tire squeezes the flow that becomes stationary in front, making the air go around it. An additional investigation on the near-wake of an isolated wheel rotating in ground contact was carried out by Robin Knowles [5]. In his study, he highlighted the following features as the main characteristics of such a wake: (1) a region of velocity deficit in the shape of an inverted T, (2) large regions of reversed flow, (3) a trailing vortex system consisting of two contra-rotating vortex pairs, and (4) the ground vortices are larger, more defined, and convected further than the upper vortices. Robin proposed that, just upstream of the contact patch, the convergence of the tire and road surfaces combine to produce a localized lateral jet on each side of the wheel. The jets deflect the freestream flow as it passes the lower shoulders of the wheel, thus modifying its effective shape. It was this deflection that generated the lower lobes and dragged the observed regions of reversed flow.

Many studies have focused on an isolated inverted wing and a wheel, yet only a few analyzed the interaction between the corresponding flow structures. Diasinos and Gatto [6] studied the effect of the angle of attack (AOA) and the span of a single-element wing on the wheel wake. Testing for a Reynolds number of $5.11 \times 10^{4}$, they observed that the lower-wing edge vortex interacted with the wake of the wheel, creating an asymmetric structure. For low wingspans, the vortex travels around the inside of the wheel, while for high wingspans, the opposite occurs. Regarding the AOA, observations led to the conclusion that it defined the vortex's size, strength, and degree of movement. Van der Berg [7] and Van der Berg and Zhang [8] carried out a complete study on the interaction between a double-element wing and a wheel. They utilized a non-deformable tire to study the impact of the ride height and wheel positioning, both experimentally and numerically. In their study, they concluded that the primary downforce-limiting mechanisms were vortex breakdown and separation, while the channeling effect, allegedly enhanced by wheel rotation, was the primary downforce-enhancing mechanism. Changes in wing downforce were attributed to a combination of the wheel circulation effect, obstruction by the wheel, and dumping effects. The numerical simulations were made using a grid of more than four million cells and applying a Reynolds-averaged Navier-Stokes (RANS) simulation with a Spalart-Allmaras turbulence model. Bruckner [9] studied the interaction between a double-element wing and a wheel, giving special attention to the transient effects, neglected in previous experiments. These effects are significant given the wheel's unsteady flow characteristics. A numerical approach using a steady Reynolds-averaged NavierStokes and Detached-Eddy Simulation (DES) with additional particle image velocimetry (PIV) analysis was made in order to investigate the effect those components have on each other, varying the ride height. Simulations using the Spalart-Allmaras turbulence model overpredicted the crossflow and incorrectly predicted the wheel's wake downwash. A significant underprediction on wheel drag was also found. In contrast, DES simulations accurately captured all main flow features and more precisely predicted wheel drag. In comparison to the isolated case, the downforce produced by the wing was smaller for higher ride heights and bigger with less ground clearance. This was attributed to the position of the stagnation point on the wheel, promoting a higher pressure beneath or above the wing, depending on the ride height. It was also concluded that at lower ride heights, wheel rotation had no impact on the downforce produced. The wing position was found to impact the wheel flow structure, observing an increased drag and lift at higher ride heights. Basso, Cravero, and Marsano [10] studied the interaction between a double-element wing and a wheel, with regards to the addition of a gurney flap (GF). The GF is a small appendix installed at the trailing edge of the front wing which can bring considerable performance gains. The main difference with the addition of a GF consists in a large area of low pressure under the main wing that enhances the ground effect, causing both a redistribution of the flow, observing a flow acceleration under the base of the endplate, and a better interaction with the wheel. Downforce was seen to increase by approximately $24 \%$, paired with a limited increase in the drag force. In the absence of a GF, large recirculation bubbles on the lower part of the car were detected. Another 
interesting study is the work developed by Roberts, Correia, Finnis and Knowles [11]. While most studies regarding inverted wings in ground effect focused on the straight-line condition, their study investigated the effects of the yawed condition. The yawed condition is representative of a car operating in a crosswind or when cornering, which is when downforce is primarily required. A 50\% scale model was used in wind tunnel testing to investigate forces and flow structures, and also to validate the numerical simulations. It was found that a change in surface pressures caused: asymmetric loading of the wing; the strengthening or inhibiting of vortices, depending on their rotational sense; and a reduction in both the downforce and drag. This reduction in forces was attributed to a change in stagnation locations and a reduction in the effective diffuser angle of the wing's suction surface. Still regarding yawed conditions, Patel, Garmory, and Passmore [12] studied the effects of cornering on a multi-element wing in ground effect. A numerical validation study was performed to confirm the validity of the DES methodology used. This involved comparing numerical data with wind tunnel experimental data and PIV. Asymmetry was the general rule regarding the on-surface pressure distribution. The change in downforce generated was minimal, while drag was found to increase in the cornering condition and decrease in the fixed yaw condition. This was due to the combined effects of both a variable local yaw and a higher freestream velocity at the outboard tip, leading to modifications to the surface pressure distribution. The downstream trajectory of the vortices was also significantly altered between the straight-line and cornering cases, which were governed by the direction of the freestream flow.

The new aero regulations, previously set for 2021 but postponed to 2022 due to the global pandemic, aim to reduce the loss of performance when following another car through the change in aerodynamic design philosophy. The goal is to implement a set of changes in order to obtain more downforce from the underbody of the car, relying less on over-body aero devices such as wings. Among the changes is the declutter of the front wing, thus reducing flow complexity. These changes will, in theory, create a narrower wake behind the car, enabling closer racing and overtaking. Keeping in mind the resources at our disposal, the present work aims to study the influence of these new front wing designs on wheel wake aerodynamics. This will be accomplished by examining the effects of front wing pressure distribution through the variation of the AOA of the second flap. The computational fluid dynamics (CFD) study was divided into two sections: a flow analysis and a force analysis.

\section{Methodology}

\subsection{Geometry}

The model of the car was made from the ground up, in an attempt to replicate the prototype presented by the FIA, respecting the regulations imposed. The front wing is composed of a main plate $(c=0.253 \mathrm{~m}$ and $b=0.800 \mathrm{~m})$ and two individual flaps. The first one has $c=0.148 \mathrm{~m}$ and $b=0.786 \mathrm{~m}$, and the second one has $c=0.113 \mathrm{~m}$ and $b=0.770 \mathrm{~m}$. The second flap is the only one that can rotate around an imaginary axis, such that different angles of attack can be reached, consequently changing the pressure distribution on the wing.

In accordance with the regulations, cars use 18-inch wheels, which corresponds to a wheel measuring $720 \mathrm{~mm}$ in diameter and $370 \mathrm{~mm}$ in width. The wheel was modeled so that a contact patch for a wheel camber of $2.4^{\circ}$ was considered. Finally, the wheel rim is covered, resulting in no crossflow through the wheel. Simulations were made at a 1:1 scale, which guaranteed the results were not affected by the physical scale of the model.

Some simplifications were also introduced in order to reduce computational costs, although never neglecting or compromising the quality of results. Consequently, components such as brake ducts, wheel hubs, tire deflector, suspension, and underbody were not considered. The geometry of the model with the front wing in detail can be seen in Figure 1. 


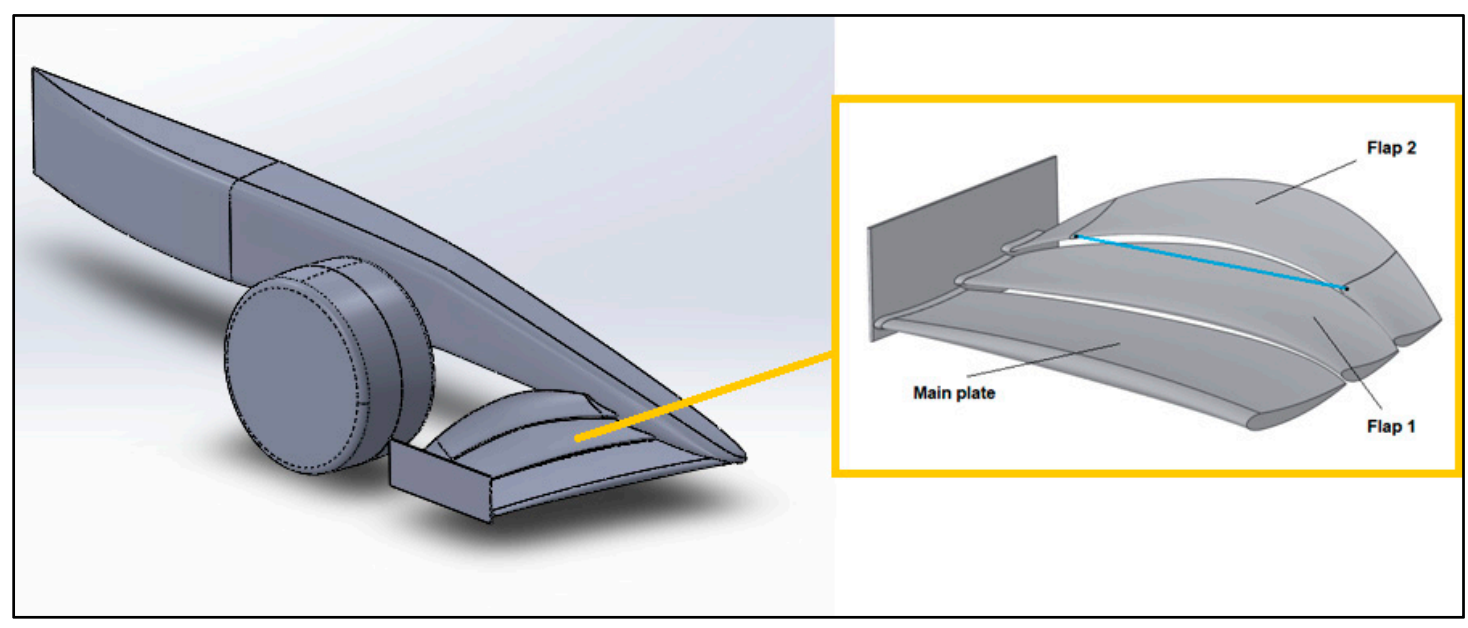

Figure 1. Geometry of the model and front wing details (blue line representing axis of rotation).

\subsection{Governing Equations}

The turbulence model used in the CFD approach was the DES model. Shur et al. [13] proposed a DES model in which the Spalart-Allmaras turbulence model is used for the unsteady RANS in the near-wall and as the subgrid-scale model for the LES in the far-wall regions. The standard Spalart-Allmaras model uses the distance to the nearest wall as the definition for the length scale $d$, which plays a major role in determining the level of production and destruction of turbulent viscosity.

The DES model replaces $d$ with a new length scale $d$ :

$$
d=\min \left(d, C_{d e s} \Delta_{\max }\right)
$$

where $C_{\text {des }}$ is an empirical constant (0.65) and $\Delta_{x i}$ is the grid spacing in the i-th direction, thus the DES resolves the averaged flow field when $d<C_{\text {des }} \Delta_{\max }$ (unsteady RANS mode) and the filtered flow field when $d>C_{\text {des }} \Delta_{\max }$ (LES mode).

For a RANS grid with a high aspect ratio in the boundary layer and where the wallparallel grid spacing usually exceeds $\delta$ (boundary layer size), the equation that defines $d$ ensures that the DES model is in the RANS mode. Nevertheless, in a case of grid ambiguity, where $\Delta<<\delta$, the DES limiter can activate the LES mode inside the boundary layer, where the grid is not fine enough to withstand resolved turbulence. Therefore, a new formulation of DES, known as the delayed option or DDES [14], is available in Ansys Fluent to preserve the RANS mode throughout the boundary layer.

The length scale $d$ is then redefined as follows:

$$
d=d-f_{d} \max \left(0, d, C_{d e s} \Delta_{\max }\right)
$$

\subsection{CFD Model}

The computational domain needs to ensure its boundaries do not disturb the flow around the car. Regarding the inlet, one needs to guarantee it does not affect the stagnation region and that it captures the entire extent of the wake. The outlet, on the other hand, cannot affect the recirculation region behind the car. Exploiting the axial symmetry of the vehicle, only one-quarter of the car was modeled - the front right corner-saving valuable computational resources. Computational domain dimensions are as follows: the inlet is located at 1.5 car lengths in front of the model, while the outlet is located at 4.5 car lengths behind it; cross section dimensions were set at $1.5 \times 1.5$ car lengths, achieving a displacement no greater than $1.5 \%$ in any given cross section along the length of the domain. Figure 2 shows the computational domain in greater detail. 


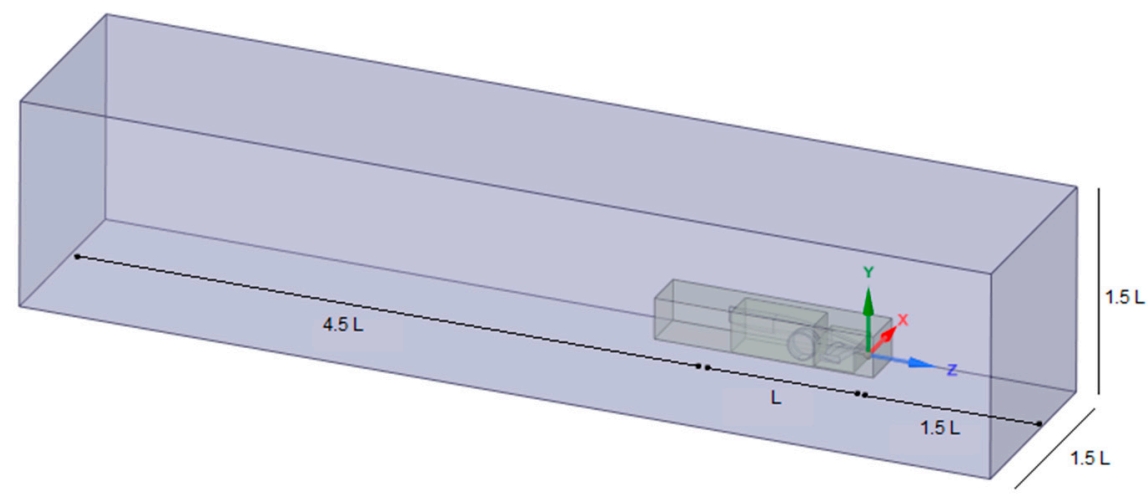

Figure 2. Computational domain dimensions.

The upstream boundary was set to "velocity-inlet" with a specified velocity of $50 \mathrm{~m} / \mathrm{s}$ (equivalent to $180 \mathrm{~km} / \mathrm{h}$ ), which is regulation defined. The downstream boundary was defined as a "pressure-outlet" with a gauge pressure of 0 . Turbulence viscosity ratio was set at $5 \%$ at the inlet and at $10 \%$ at the outlet. The symmetry plane was set as "symmetry", equivalent to a specified shear slip of zero. The top and far side walls of the domain were also given the symmetry boundary condition, keeping in mind that in order to resolve the wind tunnel boundary layer a much higher grid density had to be applied. The ground was defined as a no-slip moving wall at $50 \mathrm{~m} / \mathrm{s}$. The wheel was set as a no-slip rotating wall boundary condition, with an angular velocity of $137.9 \mathrm{rad} / \mathrm{s}$, equivalent to a tangential velocity of $50 \mathrm{~m} / \mathrm{s}$ at the contact patch. The remaining car surfaces were all set as stationary no-slip wall boundaries.

The domain needs to be discretized in order to run the necessary simulations in Ansys Fluent. A mesh independence study (ranging from 6 to 12 million cells) was performed, analyzing the effects on the values of both lift and drag coefficients. A mesh of approximately 8 million points was considered adequate given that the force coefficients values did not vary more than 1 to $2 \%$ with a higher mesh density. Accordingly, the global parameters for all sizing features were set at a growth rate of 1.2, with a minimum cell size of $1 \mathrm{~mm}$ and a maximum of $500 \mathrm{~mm}$ in order to ensure that both the geometrical attributes were well captured and a good cell quality was achieved, while limiting computational costs.

In order to properly solve the boundary layer, and aiming at a y+ value between 1-10 for the wing surface, three individual scoped prism layers were created: one for the wing, one for the wheel and another for the ground. The wing prism layer used a first cell height of $0.05 \mathrm{~mm}, 20$ layers, and a growth rate of 1.2. Ground and wheel prism layers used a first aspect ratio of 3 , with three layers and a growth rate of 1.2. The mesh can be seen in greater detail in Figures 3 and 4.

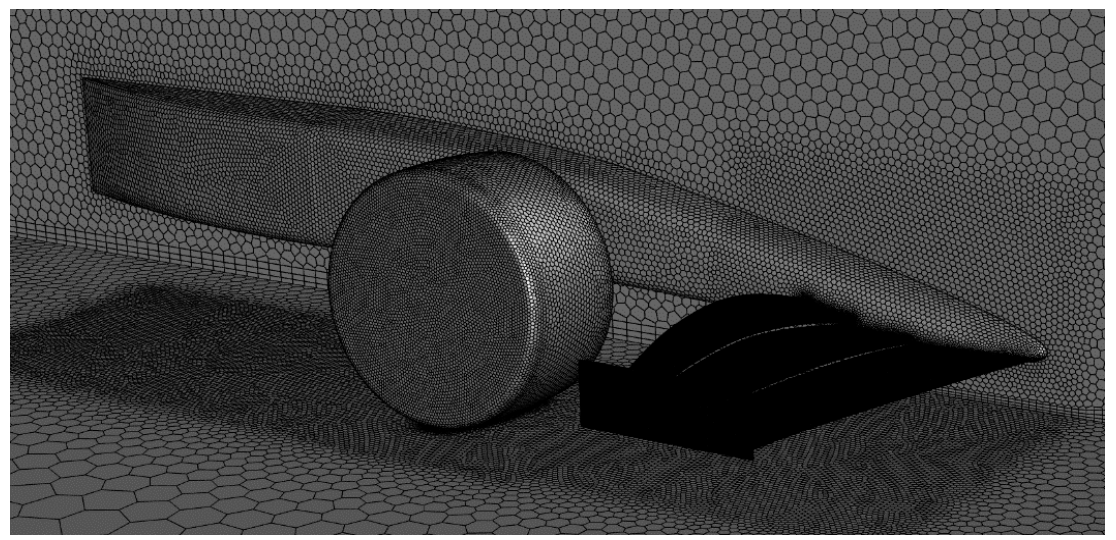

Figure 3. Mesh overview. 


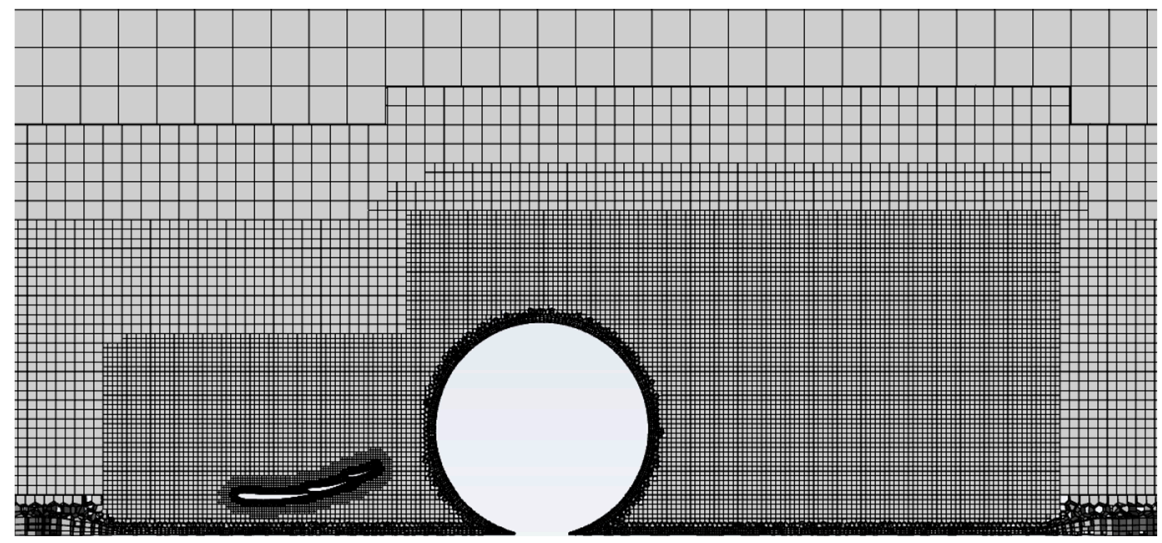

Figure 4. Side view of the grid for a plane cutting the wing and wheel.

For all the simulations carried out, a transient, pressure-based solver was used. The flow was considered isothermal and incompressible, hence no thermally-induced viscous effects were accounted for. The flow was initialized using a hybrid initialization and seeing inlet conditions as a reference to compute from. Concerning solution methods, pressure-velocity coupling was achieved through the coupled algorithm; the interpolation of pressure, momentum, and turbulent viscosity used a second-order upwind scheme. As for the transient formulation, the bounded central differencing scheme was used, in accordance with what is recommended for the DES model by the Ansys software.

In order to validate the numerical approach, a pressure coefficient study was performed by plotting and comparing the theoretical and numerical curves for a generic airfoil (NACA-2214). The objective was to evaluate the pressure coefficient obtained in Fluent and then compare it to the theoretical value (XFLR5). The experiment was carried out under the same numerical approach as the simulations: mesh parameters, turbulence model, discretization scheme, etc. A representation of the computational domain and mesh can be seen in Figure 5, where $c$ refers to the wing chord. The pressure coefficient plot for both the theoretical and numerical curves is shown in Figure 6.
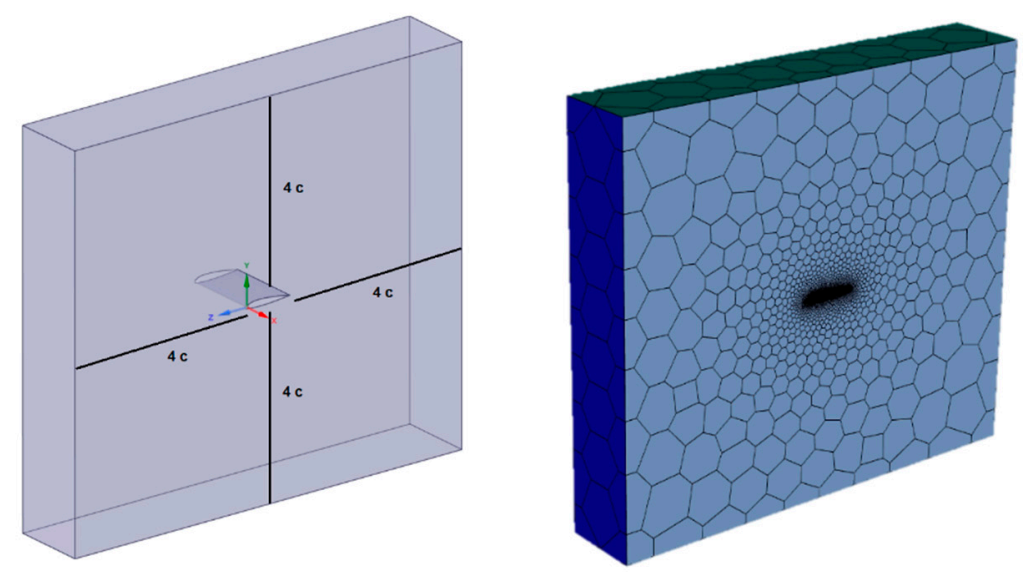

Figure 5. Computational domain and mesh overview. 


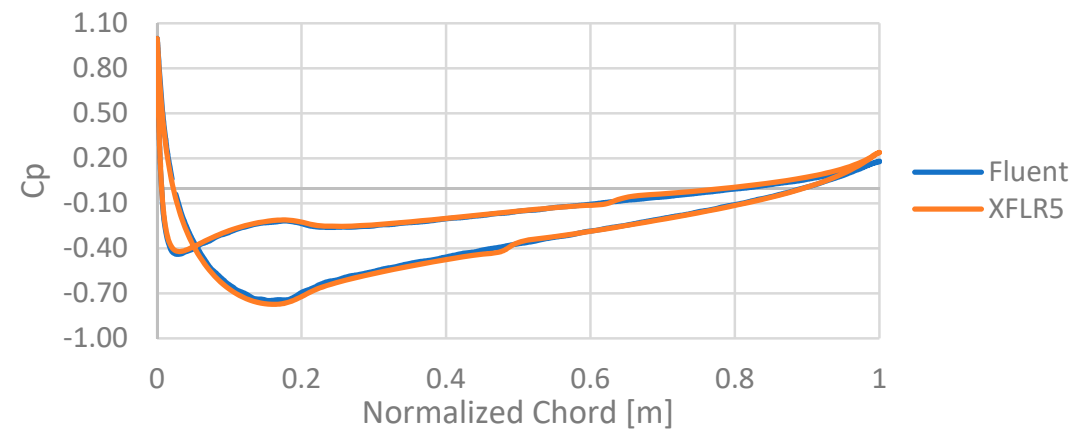

Figure 6. Pressure coefficient plot for numerical validation.

\section{Results}

\subsection{Flow Analysis}

The velocities were non-dimensionalized by the freestream velocity $(U=50 \mathrm{~m} / \mathrm{s})$, giving a more comprehensive look at the imagery extracted from Fluent. Visualizing the behavior of the flow is imperative in order to reach any conclusions about the wing configuration. For this reason, several measurement planes were placed along the length of the car, as shown in Figure 7.

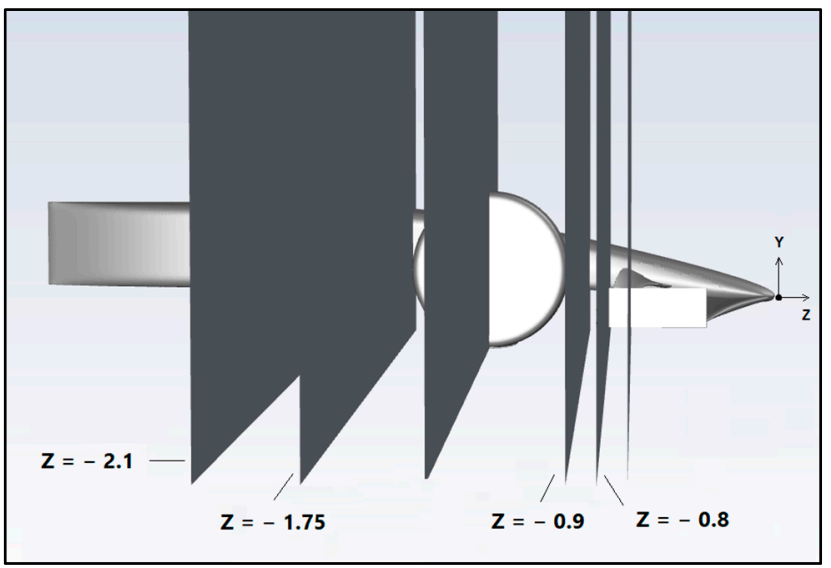

Figure 7. Measurement planes representation.

In Formula One, it is common practice to examine the complex flow structures with a total pressure coefficient $\left(C_{P} T\right)$ analysis. $C_{P} T$ is a non-dimensional parameter which translates the energy of the flow relative to the car. The red areas indicate a high energy flow, while the blue areas indicate a low energy flow, making it possible to extract some information about how effective the components will be and where there is still high energy flow to play with.

The streamwise and vertical velocity parameters offer a great illustration of the wake of the wheel. The streamwise velocity displays the velocity deficit caused by the reduced dynamic pressure in the wheel wake, while the vertical shows the change in flow angle, where zero is aligned with the freestream flow, positive is upwash, and negative is downwash.

\subsubsection{Wing}

Analyzing the $C_{P} T$ plots at plane $z=-0.8$, when compared to the other two geometries, the vortex seen for an AOA of $32^{\circ}$ is considerably smaller and, judging by the color scale, one can also conclude less energy is lost by the flow (see Figure 8). From this, one can deduce that while this vortex is still relatively cohesive, the other two geometries $\left(30^{\circ}\right.$ and $34^{\circ}$ ) see a vortex breakdown starting to occur. However, at plane $z=-0.9$, the breakdown of the lower-edge vortex is seen beneath the wing in all geometries, observing a wide 
disturbed structure, which causes recirculation (see Figure 9). This breakdown occurs as a consequence of the lower-edge vortex that is produced being too weak to sustain flow development. This has a major impact on how the wing influences the wheel flow, as only a coherent vortex can significantly influence and laterally contain the wake coming from the wheel. It remains important to mention that the AOA has little to no influence on the upper vortex. This is because the pressure increase provided by the stagnation point at the most upstream location of the wheel is greater than that which may be obtained by increasing the AOA [15].

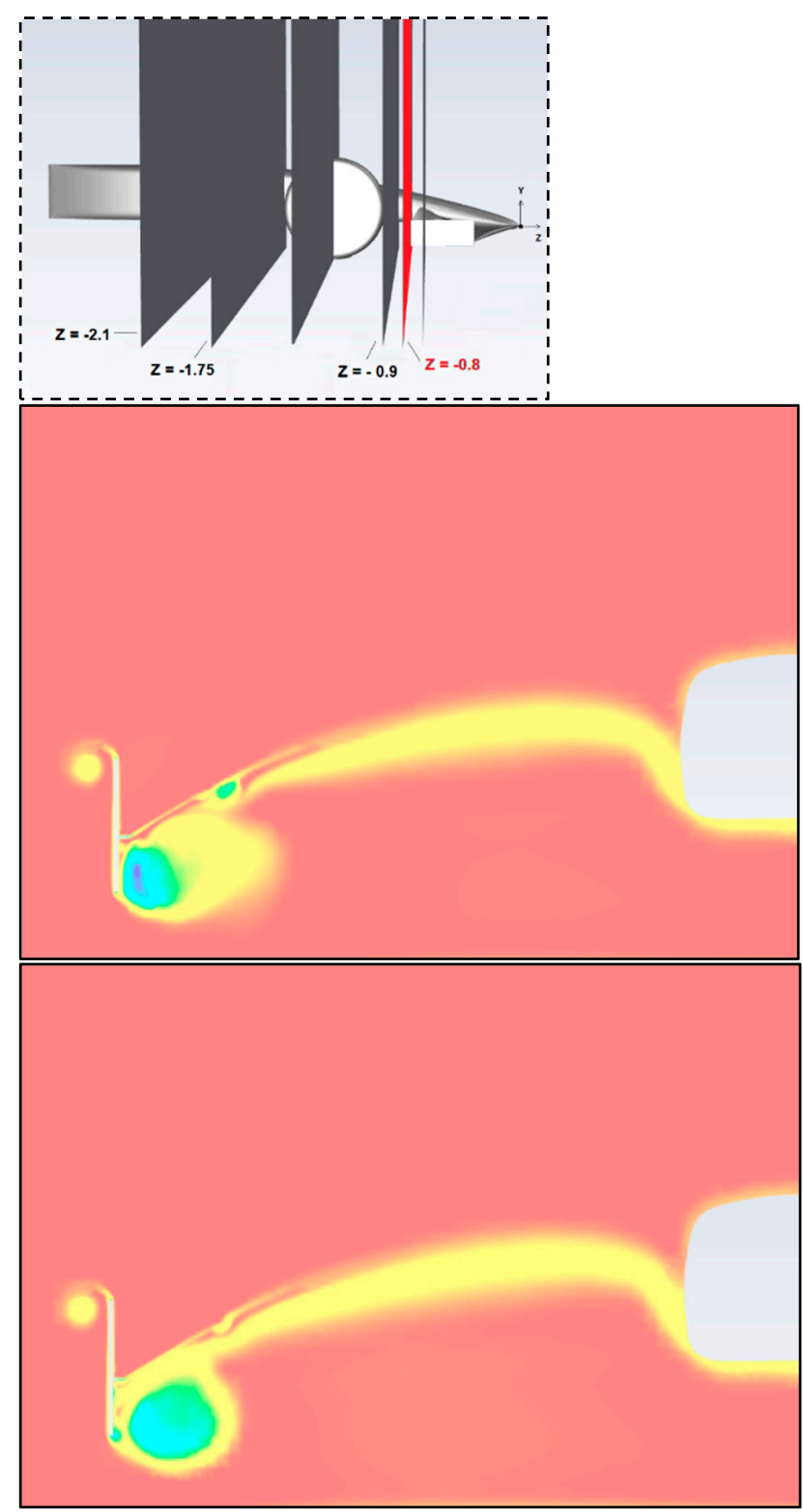

Figure 8. Cont. 


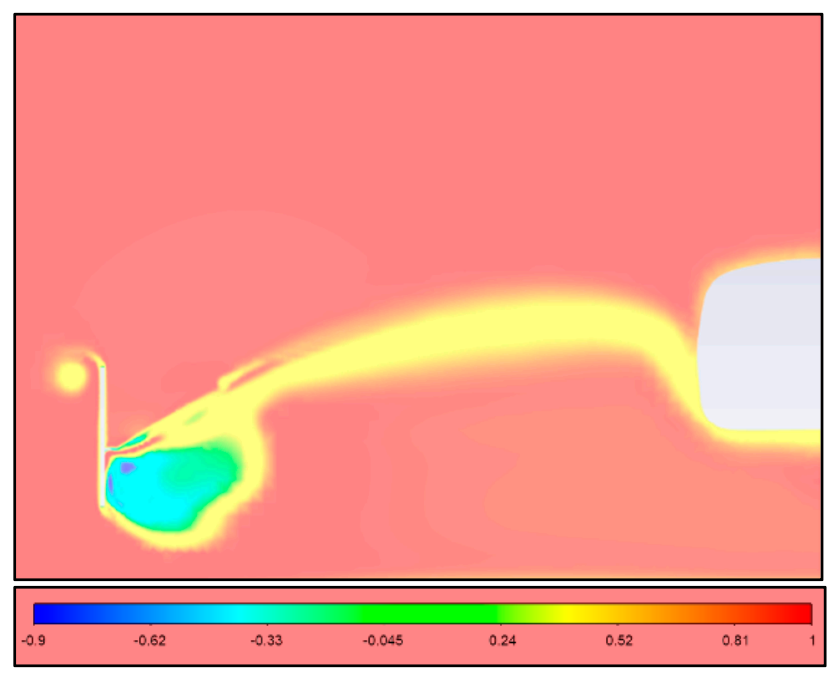

Figure 8. Total pressure coefficient at plane $\mathrm{z}=-0.8\left(\mathrm{AOA}\right.$ of $30^{\circ}, 32^{\circ}$, and $\left.34^{\circ}\right)$.
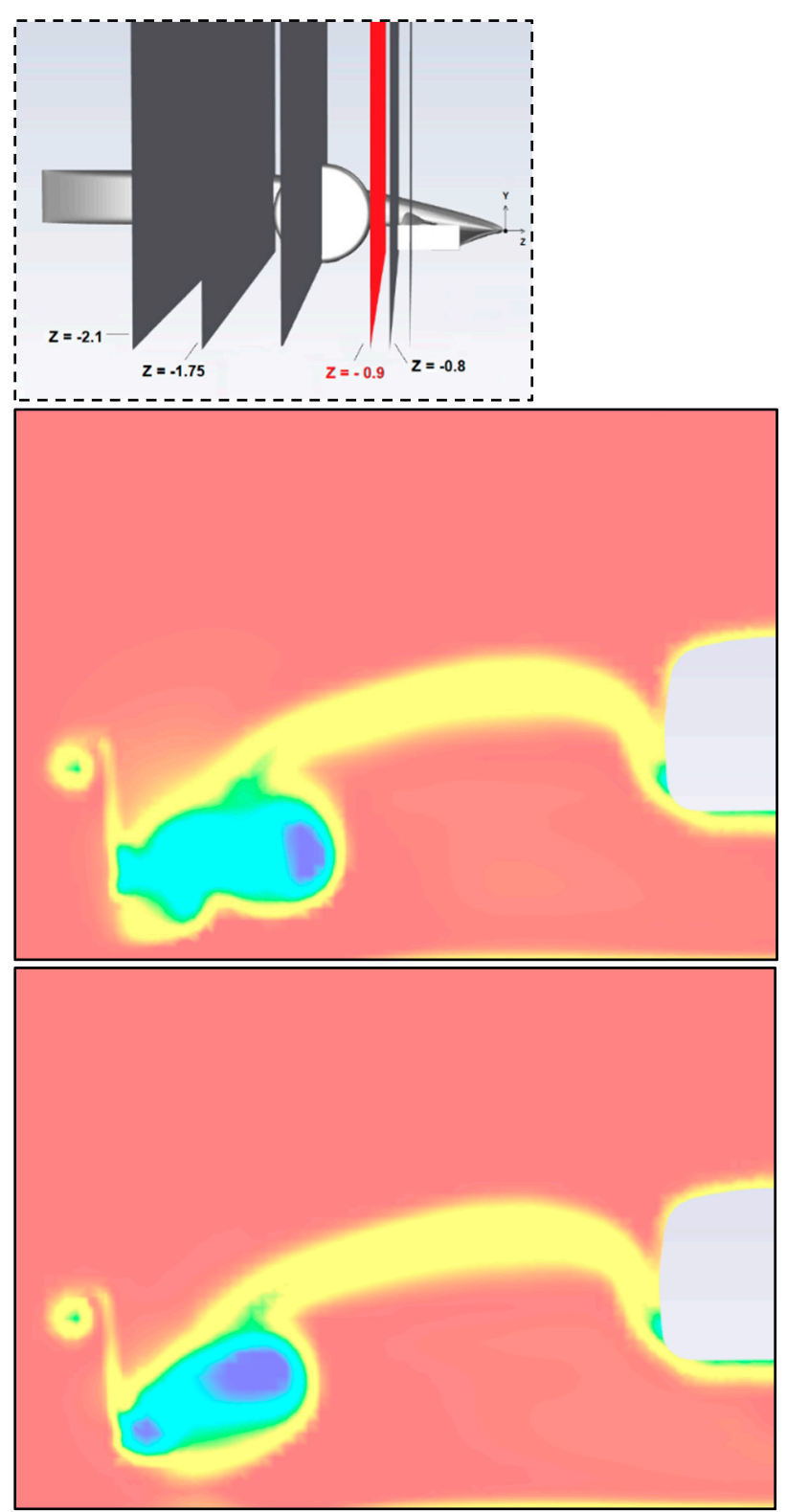

Figure 9. Cont. 


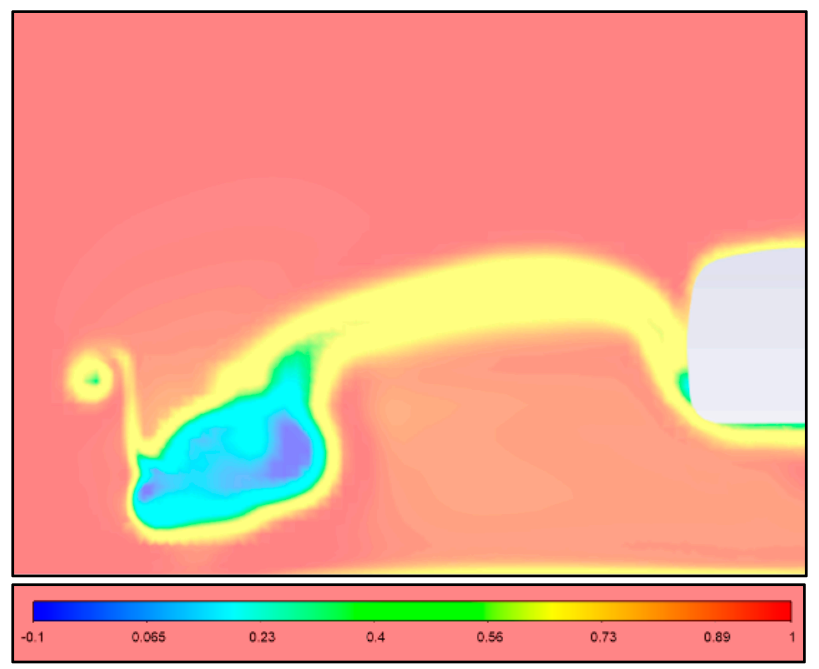

Figure 9. Total pressure coefficient at plane $\mathrm{z}=-0.9\left(\mathrm{AOA}\right.$ of $30^{\circ}, 32^{\circ}$, and $\left.34^{\circ}\right)$.

\subsubsection{Wheel}

Influenced by the flow coming from the wing, the wheel aerodynamics behave differently depending on the flap configuration, which then influences the feeding process of the underbody due to their proximity.

The wheel wake can be divided into two regions: an upper wake and a lower wake. The lower-wheel wake extends further downstream, which is explained by the ground presence and jetting effect [9]. At plane $z=-2.1$, placed where the intake of the underbody would be, the wake coming from the wheel is generally around the same size for both the $30^{\circ}$ and $32^{\circ}$ configurations. That said, the latter sees the lower wake more to the side, disrupting the area of intake more (see Figure 10 ). With regards to the $34^{\circ}$ configuration, a wider but significantly shorter wake structure, which is also located closer to the chassis, can be observed. This structure displays more areas of low energy flow, indicating more energy is lost in comparison to the other two cases.

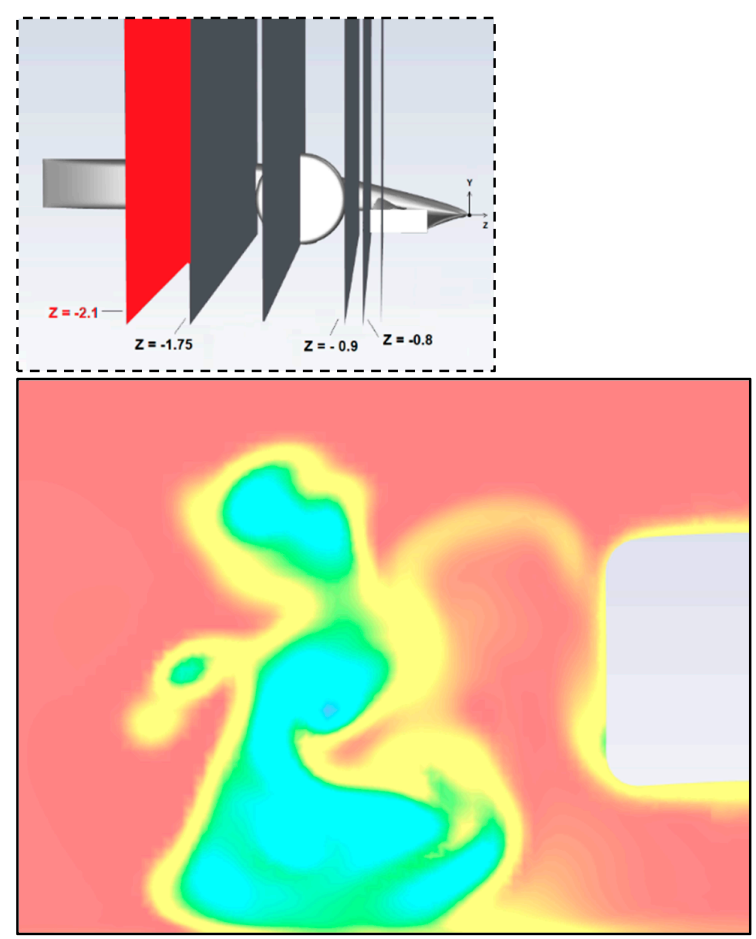

Figure 10. Cont. 


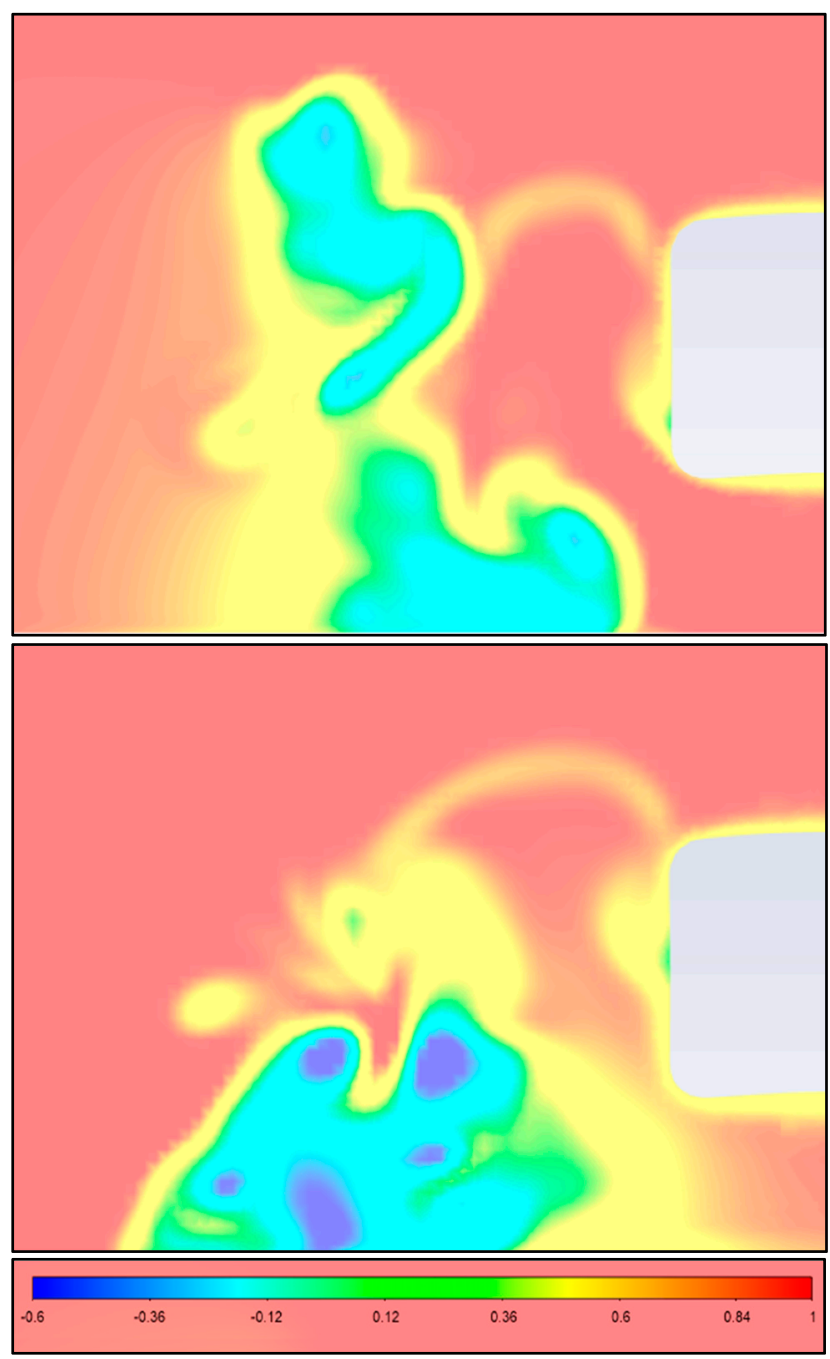

Figure 10. Total pressure coefficient at plane $\mathrm{z}=-2.1$ (underbody intake) (AOA of $30^{\circ}, 32^{\circ}$, and $34^{\circ}$ ).

Looking at the streamwise velocity at plane $z=-1.75$ (behind the wheel), the wake shows a very similar structure between the $30^{\circ}$ and $32^{\circ}$ configurations (see Figure 11 ), both exhibiting areas of strong reversed flow that are contained within the profile of the tire. The same cannot be said about the $34^{\circ}$ configuration, where the wake is dissimilar, being shorter but wider at ground level, resembling more an inverted $\mathrm{T}$ shape, as observed by Knowles [5]. In this configuration, there are no concentrated zones of strong flow reversal, unlike the other two geometries. The explanation for this may reside on the upper wake structure being stronger, which entrains more flow into this region, thus replacing in-wash (reversed flow) with downwash [16].

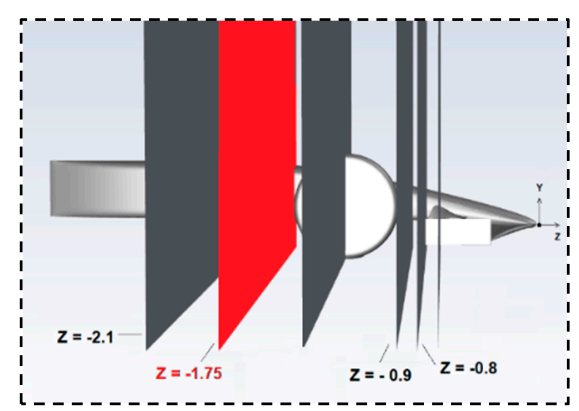

Figure 11. Cont. 


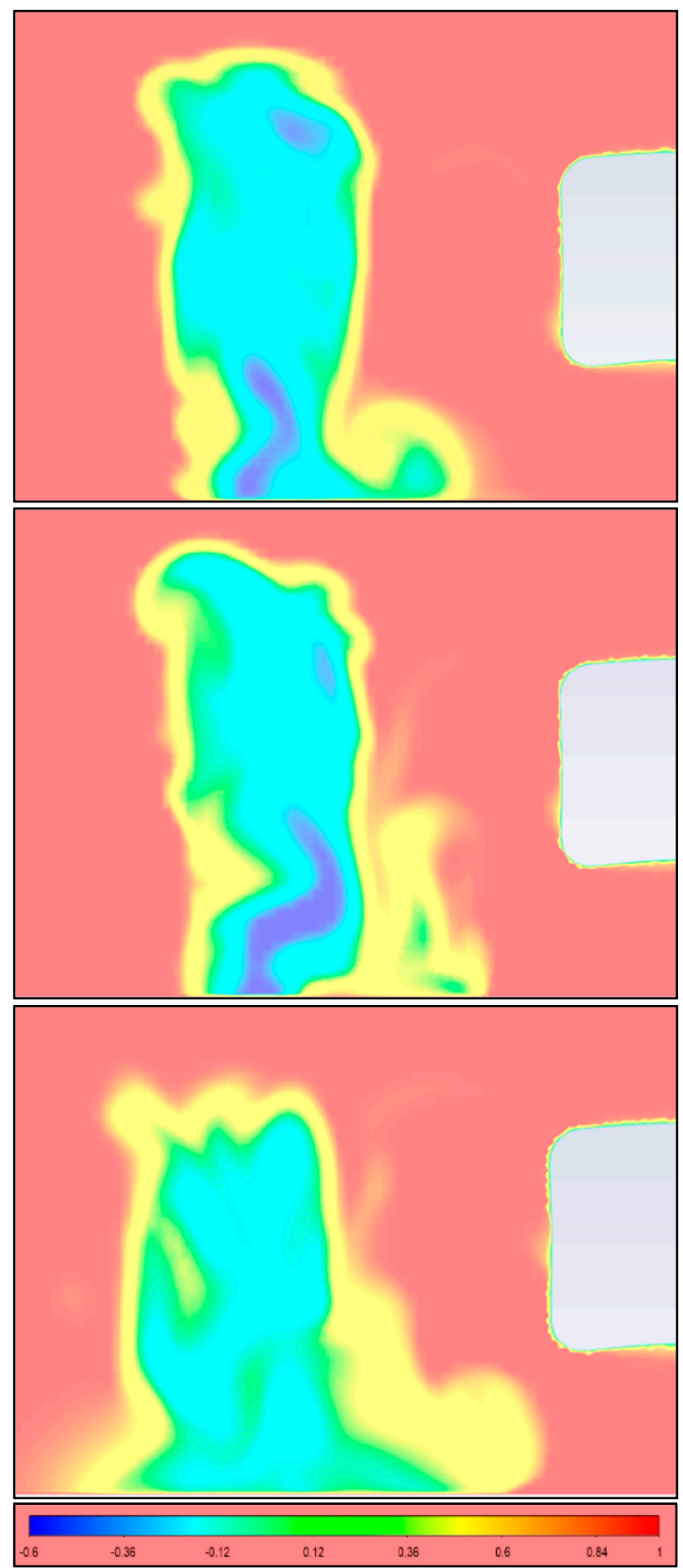

Figure 11. Streamwise velocity at plane $\mathrm{z}=-1.75$ (behind the wheel) $\left(\mathrm{AOA}\right.$ of $30^{\circ}, 32^{\circ}$, and $34^{\circ}$ ).

Further downstream, at plane $z=-2.1$ (underbody intake), a similar behavior is observed: the wake structure is again similar between the baseline and the $32^{\circ}$ geometries; however, the strong reversed flow regions are no longer present (see Figure 12). The containment of the wake structure within the profile of the tire may be an explanation for this lack of advection of the reversed flow downstream of the wheel [16]. 


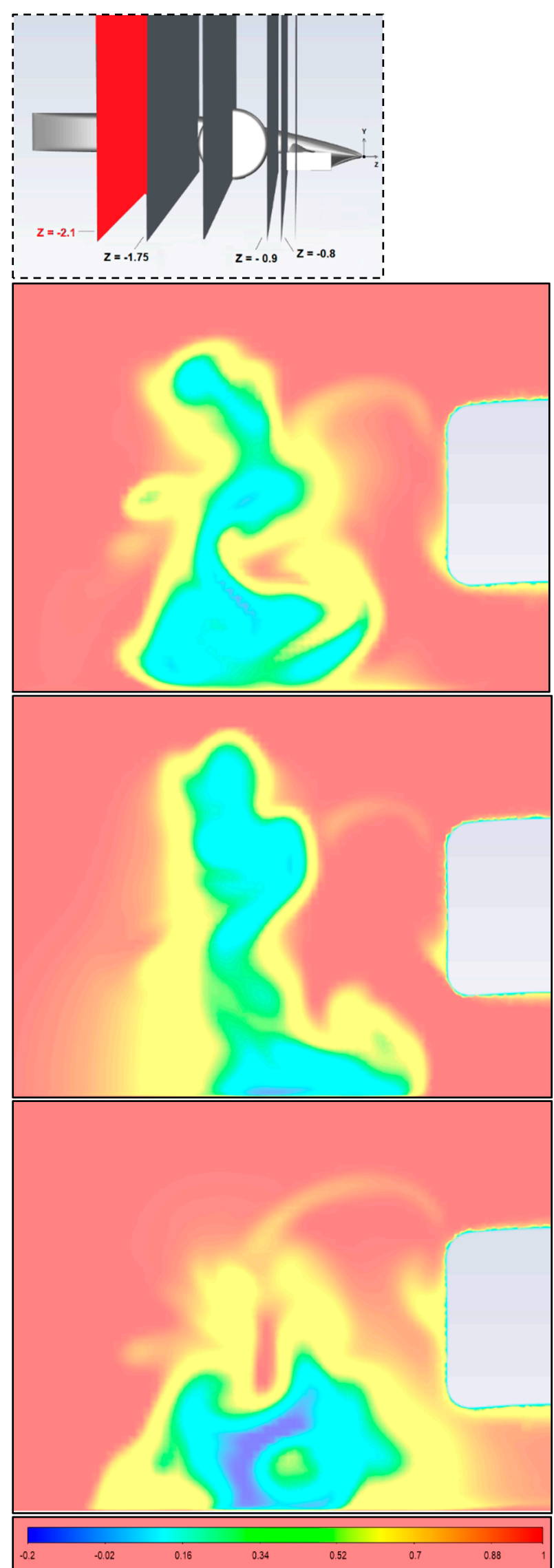

Figure 12. Streamwise velocity at plane $\mathrm{z}=-2.1$ (underbody intake) $\left(\mathrm{AOA}\right.$ of $30^{\circ}, 32^{\circ}$, and $34^{\circ}$ ). 
Regarding the vertical velocity, for a plane $z=-1.75$ (behind the wheel), overall, one can observe a very similar wake structure between the AOA of $32^{\circ}$ and the baseline geometries (see Figure 13). With regards to the AOA of $34^{\circ}$ configuration, the wake is rather different, observing a fairly large area of downwash behind the central region of the wheel (for a vertical plane). This observation goes to justify what was seen in the streamwise velocity analysis, because as the separation point moves rearwards, the downwash generated in the central region of the wheel wake increases incrementally, leading to a resultant wake that is closer to the ground (shorter) and further apart [15]. The delay of the separation point was attributed to the more aggressive flap configuration (AOA of $34^{\circ}$ ) inducing more upwash, thus providing the boundary layer with more energy to overcome the adverse pressure region on top of the tire. The increased downwash experienced in the central region of the wheel is also the explanation as to why more areas of low energy flow were seen in Figure 10, regarding the $34^{\circ}$ case; in other words, why more drag is produced by the wheel [15].

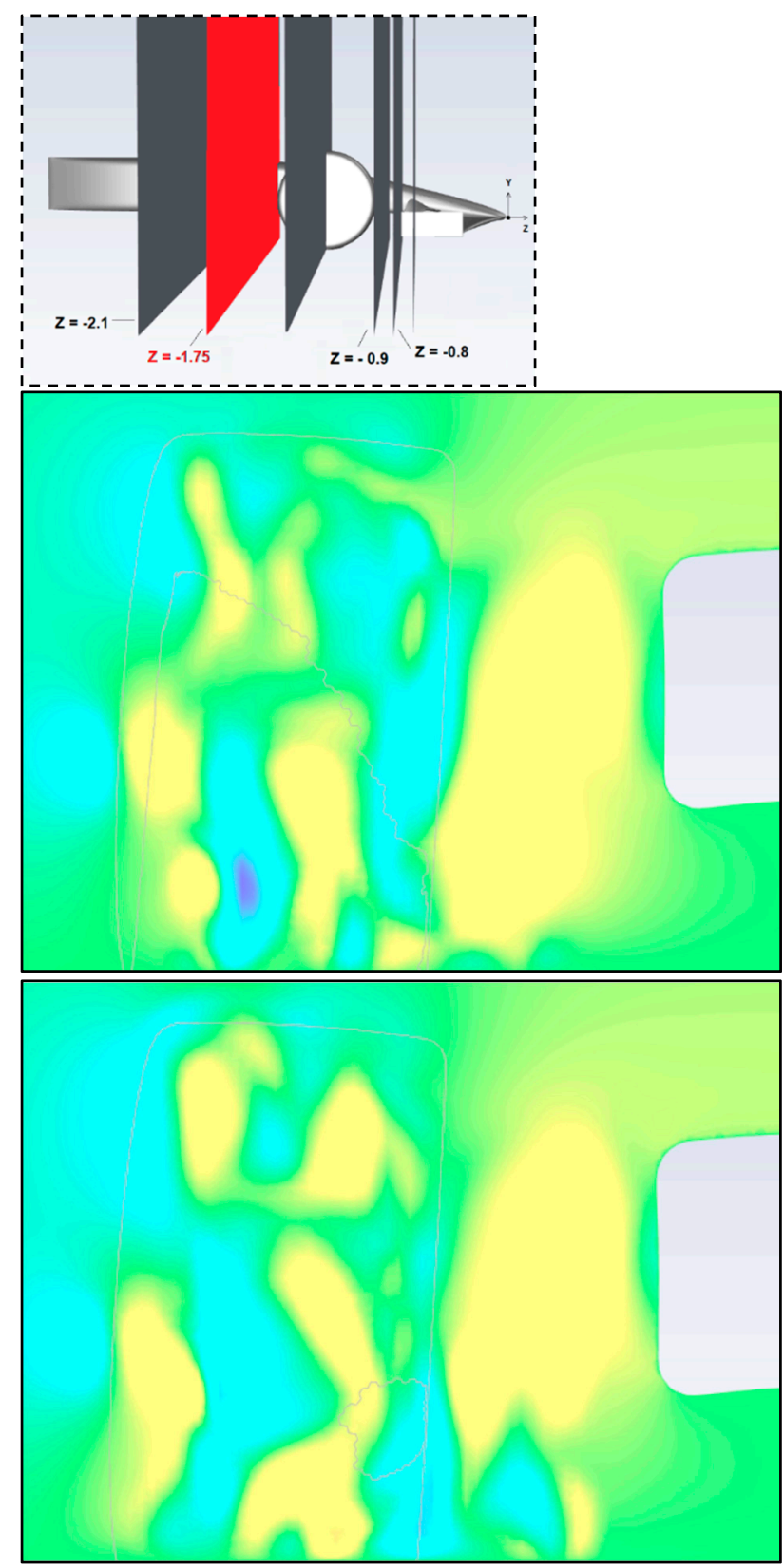

Figure 13. Cont. 


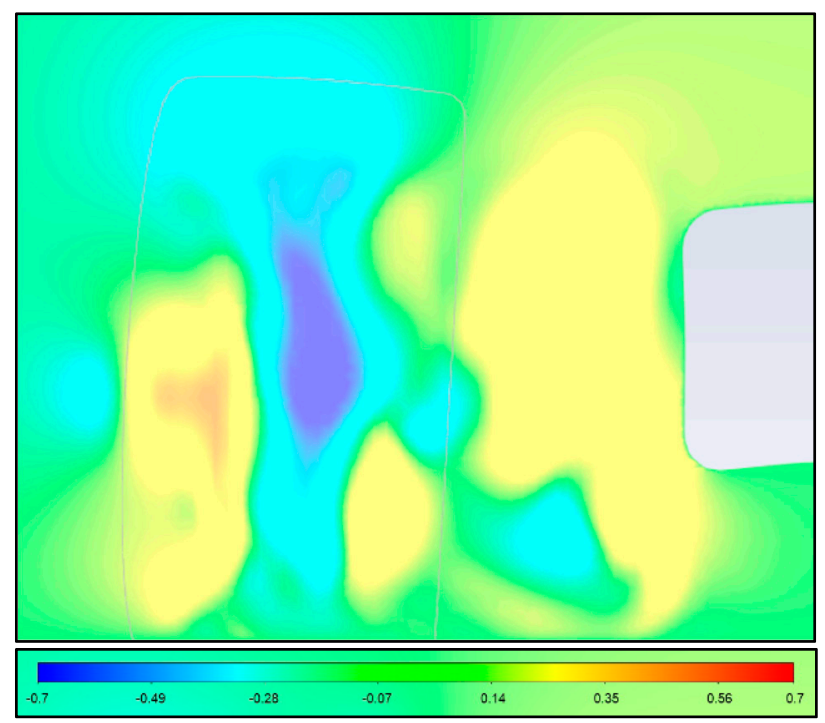

Figure 13. Vertical velocity at plane $\mathrm{z}=-1.75$ (behind the wheel) $\left(\mathrm{AOA}\right.$ of $30^{\circ}, 32^{\circ}$, and $34^{\circ}$ ).

\subsection{Force Analysis}

When it comes to unsteady simulations, oscillations of the force coefficients are foreseeable. Investigating these transient variations provides a good picture at how the unsteadiness of the lower-edge vortex influences the performance of the wing. The oscillations were examined using a fast Fourier transform (FFT) - a mathematical method used to transform a function of time into a function of frequency. The resulting plots allow one to inspect if there are any dominant frequencies, and they can be seen in Figure 14. The oscillations and dominant frequencies detected are summarized in Table 1. Seeing that the vortex breakdown is located near the wing's trailing edge might explain why higher drag force fluctuations are observed, relative to downforce fluctuations, in all three configurations [9]. The Reynolds number translates the relative importance of viscous versus pressure forces. At high Reynolds numbers such as this study, pressure drag dominates, so the most effective way of reducing drag is to reduce the size of the low-pressure wake [17]. Therefore, observing a large recirculation zone at the trailing edge of the wing is synonymous with a low-pressure region wake and, consequently, increased pressure drag. 

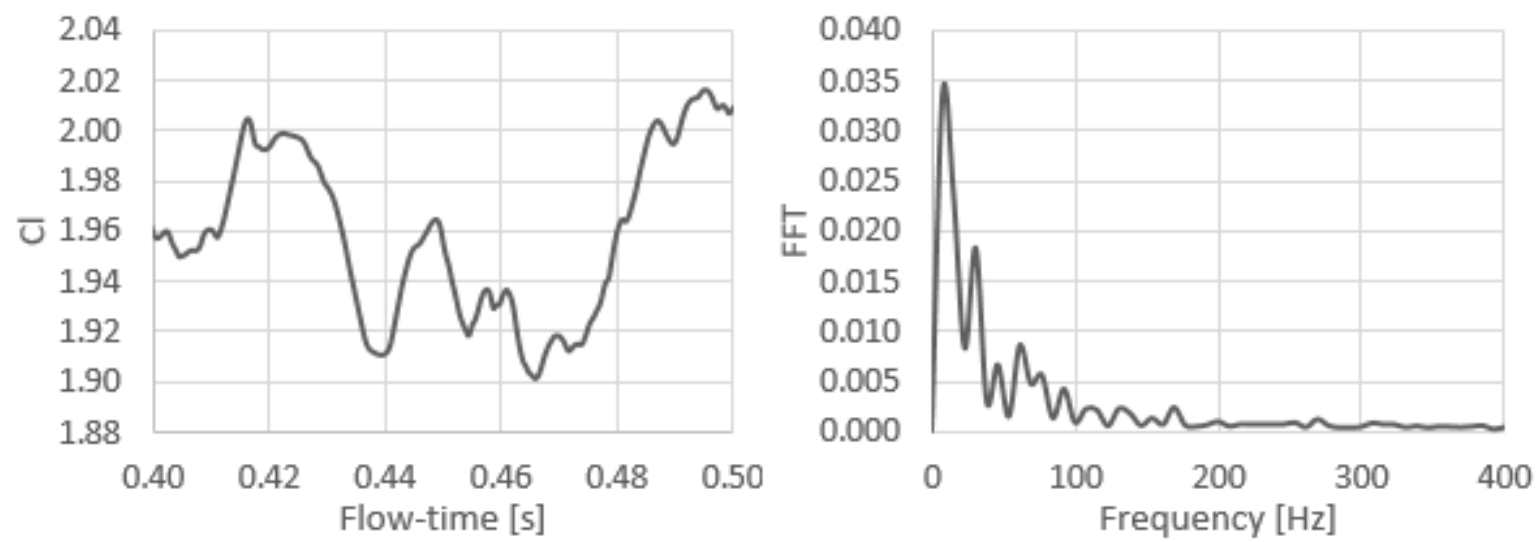

(a)
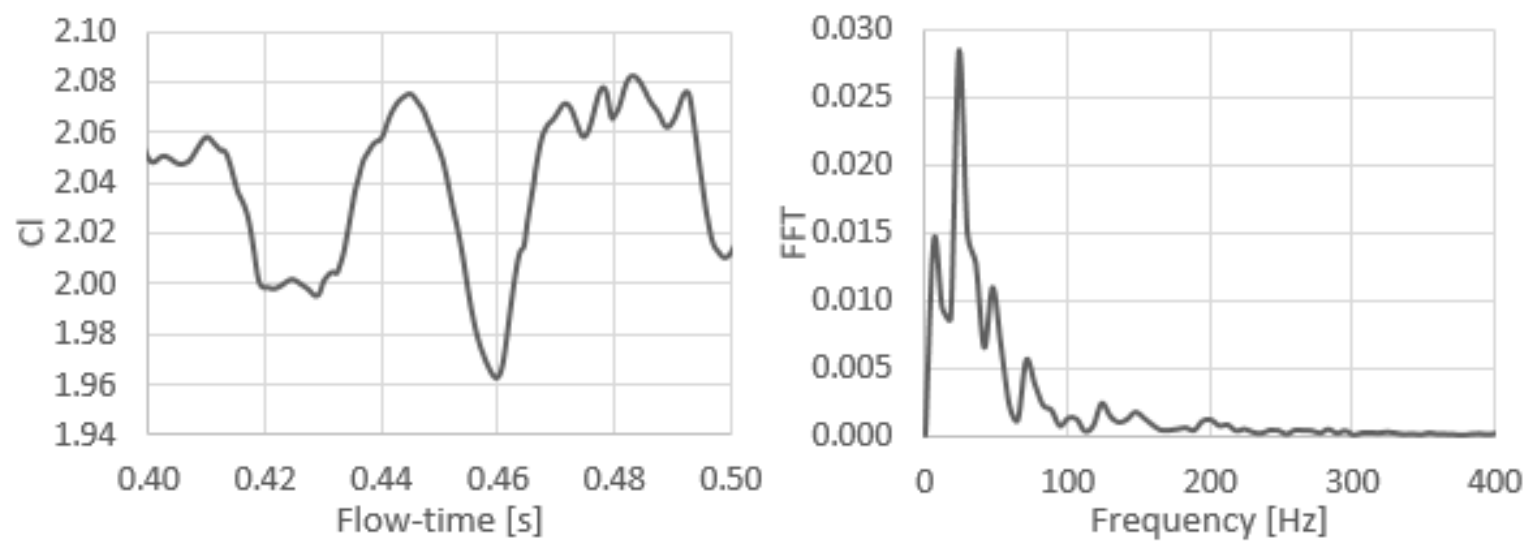

(b)
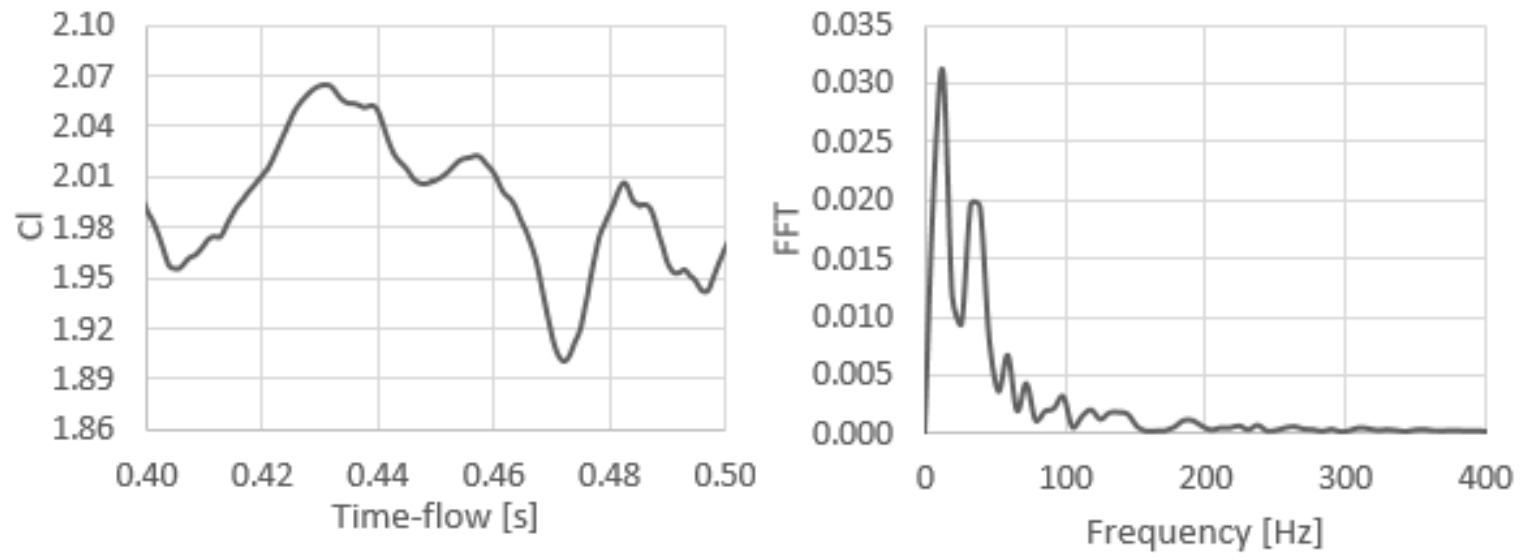

(c)

Figure 14. Oscillations with time and fast Fourier transform of the wing downforce coefficient for an $\mathrm{AOA}$ of $(\mathbf{a}) 30^{\circ}$, (b) $32^{\circ}$, and (c) $34^{\circ}$. 
Table 1. Oscillations and dominant frequencies with flap configuration.

\begin{tabular}{cccc}
\hline Flap Configuration & $30^{\circ}$ & $32^{\circ}$ & $34^{\circ}$ \\
\hline$C_{L}$ & -3 to $+3 \%$ & -4.5 to $+1.3 \%$ & -3 to $+5 \%$ \\
\hline Dominant Frequency & $8 \mathrm{~Hz}$ & $24 \mathrm{~Hz}$ & $13 \mathrm{~Hz}, 36 \mathrm{~Hz}$ \\
\hline$C_{D}$ & -4.5 to $+3.5 \%$ & -8 to $+4 \%$ & -7 to $+4.5 \%$ \\
\hline
\end{tabular}

\title{
4. Conclusions
}

As one of the primary downforce-limiting mechanisms, the vortex breakdown occurring underneath the wing has considerable repercussions on its performance. Further downstream, wheel wake management is also impaired, as only a coherent vortex could significantly influence and laterally contain the wake coming from the wheel. The pressure increase provided by the stagnation point at the most upstream location of the wheel is greater than that which may be obtained by increasing the AOA, explaining why the flap configuration has little to no influence on the upper-edge vortex. When comparing the upper- and lower-wheel wake, the latter extends further downstream, which is explained by the ground presence and jetting effect. As the separation point on top of the tire moves rearwards, the downwash increases incrementally, which explains the higher wheel drag and also leads to a resultant wheel wake that is shorter and further apart. The delay of the separation point is a consequence of the increased upwash produced by the more aggressive flap configurations. The strong regions of reversed flow observed behind the wheel were no longer present further downstream. The containment of the wake structure within the profile of the tire may be the explanation for this lack of advection, regarding the reversed flow. Finally, when compared to the downforce fluctuations, higher drag force fluctuations are detected. This occurrence was attributed to the proximity between the region of instability (i.e., vortex breakdown) and the wing's trailing edge.

Author Contributions: Formal analysis, D.M., and J.C.; funding acquisition, A.S.; supervision, J.C. and A.S.; validation, J.C.; writing-original draft, D.M.; writing—review and editing, D.M., J.C. and A.S. All authors have read and agreed to the published version of the manuscript.

Funding: The present work was performed under the scope of activities at the Aeronautics and Astronautics Research Center (AEROG) of the Laboratório Associado em Energia, Transportes e Aeronáutica (LAETA), and was supported by the Fundação para a Ciência e Tecnologia (Project No. UIDB/50022/2020).

Institutional Review Board Statement: Not applicable.

Informed Consent Statement: Not applicable.

Data Availability Statement: Not applicable.

Conflicts of Interest: The authors declare no conflict of interest. The funders had no role in the design of the study; in the collection, analyses, or interpretation of data; in the writing of the manuscript, or in the decision to publish the results.

\author{
Abbreviations \\ AOA Angle of Attack \\ CFD Computational Fluid Dynamics \\ DES Detached-Eddy Simulation \\ FFT Fast Fourier Transform \\ FIA Federation Internationale de l'Automobile \\ GF Gurney Flap \\ LES Large Eddy Simulations \\ PIV Particle Image Velocimetry \\ RANS Reynolds-averaged Navier-Stokes
}




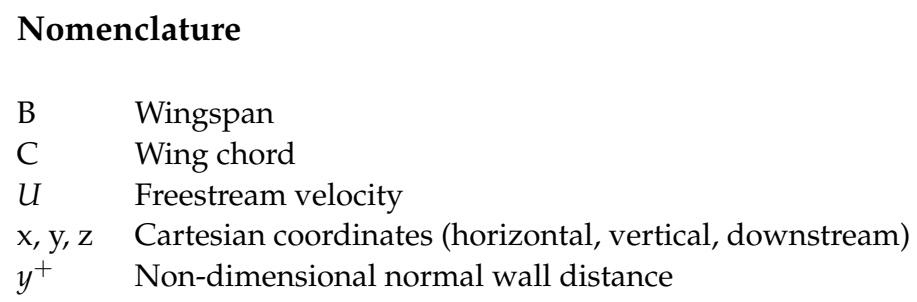

\section{References}

1. Agathangelou, B.; Gascoyne, M. Aerodynamic Design Considerations of a Formula 1 Racing Car. In SAE Technical Papers; SAE International: Warrendale, PA, USA, 1998.

2. Pegrum, J.M. Experimental Study of the Vortex System Generated by a Formula 1 Front Wing. Ph.D. Thesis, Imperial College London, London, UK, 2007.

3. Mercker, E.; Breuer, N.; Berneburg, H.; Emmelmann, H.J. On the Aerodynamic Interference Due to the Rolling Wheels of Passenger Cars. In SAE Technical Papers; SAE International: Warrendale, PA, USA, 1991.

4. Fackrell, J.E. The Aerodynamics of an Isolated Wheel Rotating in Contact with the Ground. Ph.D. Thesis, University of London, London, UK, 1974.

5. Knowles, R.D. Monoposto Racecar Wheel Aerodynamics: Investigation of Near-Wake Structure and Support-Sting Interference. Ph.D. Thesis, Cranfield University, Cranfield, UK, 2007.

6. Diasinos, S.; Gatto, A. Experimental Investigation into Wing Span and Angle-of-Attack Effects on Sub-Scale Race Car Wing/Wheel Interaction Aerodynamics. Exp. Fluids 2008, 45, 537-546. [CrossRef]

7. Van Den Berg, M.A. Aerodynamic Interaction of an Inverted Wing with a Rotating Wheel. Ph.D. Thesis, University of Southampton, Southampton, UK, 2007.

8. Van Den Berg, M.A.; Zhang, X. The Aerodynamic Interaction between an Inverted Wing and a Rotating Wheel. J. Fluids Eng. Trans. ASME 2009. [CrossRef]

9. Heyder-Bruckner, J. The Aerodynamics of an Inverted Wing and a Rotating Wheel in Ground Effect. Ph.D. Thesis, University of Southampton, Southampton, UK, 2011.

10. Basso, M.; Cravero, C.; Marsano, D. Aerodynamic Effect of the Gurney Flap on the Front Wing of a F1 Car and Flow Interactions with Car Components. Energies 2021, 14, 2059. [CrossRef]

11. Roberts, L.S.; Correia, J.; Finnis, M.V.; Knowles, K. Aerodynamic Characteristics of a Wing-and-Flap Configuration in Ground Effect and Yaw. Automob. Eng. 2016, 230, 841-854. [CrossRef]

12. Patel, D.; Garmory, A.; Passmore, M. The Effects of Cornering on the Aerodynamics of a Multi-Element Wing in Ground Effect. Fluids 2021, 6, 3. [CrossRef]

13. Shur, M.; Spalart, P.R.; Strelets, M.; Travin, A. Detached-Eddy Simulation of an Airfoil at High Angle of Attack. In 4th International Symposium on Engineering Turbulence Modelling and Experiments; Elsevier Science Ltd.: Amsterdam, The Netherlands, 1999.

14. Spalart, P.R.; Deck, S.; Shur, M.L.; Squires, K.D.; Strelets, M.K.; Travin, A. A New Version of Detached-Eddy Simulation, Resistant to Ambiguous Grid Densities. Theor. Comput. Fluid Dyn. 2006, 20, 181-195. [CrossRef]

15. Diasinos, S. The Aerodynamic Interaction of a Rotating Wheel and a Downforce Producing Wing in Ground Effect. Ph.D. Thesis, University of New South Wales, Sydney, Australia, 2009.

16. Knowles, R.D.; Saddington, A.J.; Knowles, K. On the near Wake of a Formula One Front Wheel. Proc. Inst. Mech. Eng. Part D J. Automob. Eng. 2013, 227, 1491-1502. [CrossRef]

17. Alexander, D.E. Fluid Biomechanics. In Nature's Machines; Academic Press: Cambridge, MA, USA, 2017. 